\title{
Die Pneumologie - ein Schwerpunktfach der Inneren Medizin
}

\author{
Pneumology - A Major Specialty of Internal Medicine
}

Autor

R. Loddenkemper

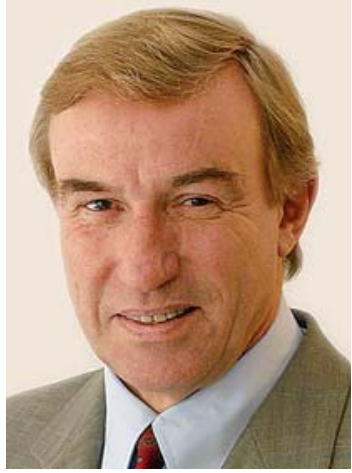

Prof. Dr. Dr. h.c. Robert Loddenkemper

\section{Bibliografie}

DOI http://dx.doi.org/ 10.1055/s-0030-1255652 Pneumologie 2010; 64: 531-532 @ Georg Thieme Verlag KG Stuttgart · New York ISSN 0934-8387

Korrespondenzadresse

Prof. Dr. Dr. h.c.

Robert Loddenkemper

Hauptstadtbüro der DGP

Stralauer Platz 34

10243 Berlin

rloddenkemper@

dzk-tuberkulose.de
Die Deutsche Gesellschaft für Pneumologie und Beatmungsmedizin (DGP) feiert in diesem Jahr ihren 100. Geburtstag. Noch in der Kaiserzeit gegründet und in der Weimarer Republik erweitert, erfuhr die Gesellschaft Gleichschaltung und Niedergang im Dritten Reich, Auflösung bei Kriegsende, Wiedergründung nach dem Zweiten Weltkrieg, separate Gründung in der DDR 1957, Wiedervereinigung nach dem Mauerfall und in den folgenden 20 Jahren ein allmähliches Aufblühen mit einer fast Verdreifachung der Mitgliederzahlen von 1000 auf 2800. Die Geschichte der DGP wird in einer umfangreichen Chronik geschildert [1].

In den zurückliegenden 100 Jahren vollzog sich die Entwicklung der heutigen DGP von der Vereinigung der Lungenheilanstaltsärzte hin zu der wissenschaftlichen Gesellschaft, die die vielfältigen Facetten der modernen Pneumologie repräsentiert. Standen die ersten 50 Jahre noch ganz im Zeichen der damals führenden Volkskrankheit Tuberkulose, so vollzog sich nach deren erfolgreicher Eindämmung in Deutschland in den letzten 50 Jahren ein erheblicher Wandel im Spektrum der Krankheiten: heute zählen Asthma, COPD und Pneumonie, inzwischen auch die Schlafapnoe, zu den großen Volkskrankheiten. Der Lungenkrebs ist führend unter den tumorbedingten Todesursachen, zusammen mit der COPD ist er für gut die Hälfte (53600 von 106300) der tabakassoziierten Todesfälle in Deutschland verantwortlich [2].

Auch andere Risikofaktoren wie Infektionserreger, Allergene, berufliche Noxen, Luftverschmutzung und Ernährung spielen eine wichtige Rolle in der Entstehung von Atemwegs- und Lungenkrankheiten. Bei Kindern ist Asthma die führende chronische Erkrankung, die Mukoviszidose (Zystische Fibrose) ist in Deutschland die häufigste Erbkrankheit.
In dieser Ausgabe der „Pneumologie“ werden anlässlich der Geburtstagsfeier der DGP am 6. Oktober in Berlin die wichtigsten pneumologischen Krankheiten komprimiert dargestellt. Dazu werden vorhandene Defizite in ihrer Bekämpfung sowie Zukunftsperspektiven aufgezeigt.

Der Artikel aus dem Institut für Krebsepidemiologie an der Universität zu Lübeck und dem Institut für Gesundheits-Systemforschung in Kiel, „Erkrankungszahlen in der Pneumologie - eine Projektion bis 2060“, der sich speziell mit fünf pneumologischen Krankheiten (Asthma, Tuberkulose, COPD, Lungenkrebs und ambulant erworbene Pneumonie) befasst [3], sagt aufgrund der demografischen Veränderungen den stärksten Anstieg (188\%) in den nächsten Jahren für die ambulant erworbene Pneumonie (CAP) voraus. Aber auch bei den vier anderen Krankheitsbildern werden zum Teil deutliche Steigerungen relativ zur Einwohnerzahl für die nächsten Jahrzehnte prognostiziert. Daher ist zu erwarten, dass die Bedeutung der Pneumologie in Zukunft zunehmen wird. Schon heute gehört sie neben der Kardiologie und der Gastroenterologie zu den drei großen Schwerpunktfächern der Inneren Medizin.

Rasante Fortschritte auf dem Gebiet der Lungenund Atemwegserkrankungen wurden nicht nur in der Diagnostik (Bildgebung, Endoskopie, molekulare Verfahren in der Mikrobiologie und Pathologie) erzielt, sondern auch auf therapeutischem Gebiet. Dies schildern die Artikel zur pulmonalen Hypertonie, zu den schlafassoziierten Ventilationsstörungen sowie zu den verschiedenen Formen der respiratorischen Insuffizienz. Bei der akuten respiratorischen Insuffizienz spielt die Intensivmedizin mit Respiratorbeatmung eine zunehmend wichtige Rolle, wobei für die Entwöhnung vom Respirator („Weaning“) derzeit ein Netzwerk innerhalb der DGP etabliert wird. Die Prognose der chronischen respiratorischen Insuf- 
fizienz verschiedenster Ätiologie ist heute nicht nur dank der Sauerstoff-Langzeittherapie, sondern besonders durch die Heimbeatmung (außerklinische Beatmung) und durch die Lungentransplantation deutlich günstiger geworden.

Welch große Bedeutung den Lungenkrankheiten zukommt, wurde schon im „Weißbuch Lunge 2005“ für Deutschland herausgestellt [4]. Unter den Arbeitsunfähigkeitsfällen lagen sie mit 25\% ebenso wie bei den Berufskrankheiten mit 59\% an der Spitze, bei der Mortalität haben sie mit 10\% einen wesentlichen Anteil, bei den Krankenhaustagen sind sie mit 7\% eher unterschätzt. Diese statistischen Zahlen spiegeln sich auch in den Kosten und damit in der sozioökonomischen Bedeutung - wider: 2002 verursachten Lungenkrankheiten (ohne Intensivmedizin) 24 Milliarden Euro und wurden nur von den Herz-Kreislauf-Krankheiten (38 Milliarden Euro) übertroffen.

Aus diesen Zahlen leitet sich auch die Forderung der Pneumologie nach besserer Repräsentanz an den Universitäten und den grundlagenorientierten Forschungseinrichtungen ab. Im Vergleich mit anderen europäischen Ländern gehört Deutschland bei der Anzahl selbstständiger pneumologischer Universitätsabteilungen zu den Schlusslichtern [5]. Historisch erklärt sich dies vor allem damit, dass sich das Fach bis in die zweite Hälfte des vo- rigen Jahrhunderts auf die Tuberkulose konzentrierte, die nahezu ausschließlich außerhalb der Universitäten versorgt wurde. In den meisten westeuropäischen Ländern und in den U.S.A. hat jede Universitätsklinik eine selbstständige pneumologische Abteilung (oft verbunden mit der internistischen Intensivmedizin). Da den Universitäten eine Schlüsselrolle in der Ausbildung zukünftiger Arztgenerationen und in der Forschung zukommt, sollte auch in Deutschland an allen Universitätskliniken eine solche Vertretung der Pneumologie gesichert werden.

\section{Literatur}

1 Deutsche Gesellschaft für Pneumologie und Beatmungsmedizin. 100 Jahre DGP - 100 Jahre deutsche Pneumologie. Heidelberg: Springer, 2010

2 Mons U. Tabakattributable Mortalität in Deutschland und in den deutschen Bundesländern - Berechnungen mit Daten des Mikrozensus und der Todesursachenstatistik. Gesundheitswesen 2010; epub ahead of print 14. Juni; DOI: 10.1055/s-0030-1252039

3 Pritzkuleit R, Beske F, Katalinic A. Erkrankungszahlen in der Pneumologie - eine Projektion bis 2060. Pneumologie 2010; 64: 535-540

4 Fabel H, Konietzko N. Weißbuch Lunge. Stuttgart, New York: Thieme, 2005

5 Loddenkemper R, Gibson GJ, Sibille Y. European Lung White Book - The first comprehensive survey of respiratory health in Europe. ERSJ Ltd., 2003 